\title{
International Clinical Guidelines for the Adoption of Digital Pathology: A Review of Technical Aspects
}

\author{
Marcial García-Rojo \\ Hospital de Jerez de la Frontera, Jerez de la Frontera, Cádiz, Spain
}

\section{Key Words}

Digital pathology · International guidelines $\cdot$ Technical

specification - Whole-slide image

\begin{abstract}
Digital slides, also called whole-slide images, are being evaluated to replace conventional microscopy, and several guidelines have been published. This paper reviews technical specifications of digital pathology systems that have been included in the guidelines and position papers from the Canadian Association of Pathologists, the College of American Pathologists, the American Telemedicine Association, the Digital Pathology Association, the Food and Drug Administration, the Centers for Medicare and Medicaid Services, the Centers for Disease Control and Prevention, the Society of Toxicologic Pathology, the European Commission, the Spanish Society of Anatomic Pathology, The Royal College of Pathologists and The Royal College of Pathologists of Australasia. In conclusion, most technical aspects are well covered by these guidelines, although they offer limited information regarding image quality and compression, and file formats.

(c) 2016 S. Karger AG, Basel
\end{abstract}

\section{Introduction}

Pathologists are looking at digital pathology not so much as an efficient telepathology solution but rather as an instrument to improve the quality and efficiency of their own daily clinical work beyond the use of the conventional microscope.

We consider the digital pathology concept as the integration of all health information systems, including data and images available in the workflow of a pathology department. Even those authors who have defined digital pathology as the creation, viewing, management, sharing, analysis and interpretation of digital images of glass slides (digital imaging) also include workflow considerations [1-3].

Main improvements in information technology (IT) in pathology, in the last 10 years, are those in pathology/ laboratory information systems (LIS), barcoding and tracking systems, and digital images, especially wholeslide imaging (WSI). However, WSI technology is only emerging in clinical practice, and, until now, it is mainly employed in education and research in pathology [4-6].

The Digital Pathology Resource Guide of the College of American Pathologists (CAP) includes a section dedicated to guidelines, accreditation and proficiency testing

\section{KARGER}

E-Mail karger@karger.com www.karger.com/pat
(C) 2016 S. Karger AG, Base

1015-2008/16/0833-0099\$39.50/0
Marcial García-Rojo

Hospital Jerez de la Frontera (AGS Norte de Cádiz), UGC Anatomía Patológica Ronda de Circunvalación $\mathrm{s} / \mathrm{n}$ ES-11407 Jerez de la Frontera (Spain)

E-Mail marcial@cim.es 
[1], and references to guidelines or position papers other than CAP guidelines are dispersed in other sections of this document. Recently, Hanna et al. [7] reviewed existing guidelines and position statements for digital pathology, with special attention to validation and telepathology. A recent comparison between American Telemedicine Association (ATA), Royal College of Pathologists (RCP) and Canadian telepathology guidelines is also available [8].

This paper reviews the international pathology initiatives that have been developed to understand the role that digital pathology can play in anatomic pathology and to learn how to validate or implement a digital pathology system in the clinical environment. We shall not focus on telepathology or IT standardization but on some technical aspects of best-practice guidelines that have been considered necessary to have safe on-site clinical implementation of digital pathology systems for primary diagnosis.

\section{Digital Pathology Guidelines}

The following digital pathology guidelines, comprising technical and clinical recommendations, have been published in the last 8 years. These documents have been developed to achieve safe and reliable patient care based on best practices developed from existing scientific evidence and the experience of experts in the field, i.e. early adopters of digital pathology [7]. A list of organizations and guidelines from different countries is included in table 1 of this review.

\section{Description of Guidelines}

The Digital Pathology Association (DPA) published the first validation guideline on WSI in a clinical environment in 2011. It is an overview on how to perform validation studies, the regulatory agencies involved, vendor's responsibility and quality assurance aspects [16]. Interoperability between the anatomical pathology LIS and the digital pathology systems was also reviewed by this organization [17]. The DPA guidelines make some additional technical comments on the draft of the CAP guidelines [16].

The guidelines from the CAP Pathology and Laboratory Quality Center are aimed to design a validation process before WSI is implemented for primary diagnosis in clinical use, and 12 statements ensued. These guidelines, published in 2013, reviewed the literature from 2000 through January 2012, and 23 publications covering clinical applications for WSI were selected [11]. For the first time, an evidence-based approach was used to evaluate the final recommendations included in this document.

The Anatomic Pathology Checklist is an instrument of the CAP accreditation program to summarize those requirements regarding proficiency testing, procedure manuals, test method validation and critical results [13]. The section dedicated to telepathology includes virtual slides/WSI, WSI user training and system validation components, and a digital image analysis section [1]. Some sections (i.e. procedure manual, quality management, surgical pathology reports, fluorescence and nonfluorescence in situ hybridization, digital image analysis, instruments and equipment) need to be considered when implementing digital pathology in a clinical laboratory [28].

The ATA guidelines provide a brief overview of technical specifications for telepathology, including viewing displays, transmission and storage, and a succinct list of clinical applications, responsibilities, training, quality management and operational aspects $[3,8,14,15]$. This is the only guideline to include the use of digital pathology in rapid on-site adequacy evaluation of fine-needle aspiration cytology specimens [7]. Austrian telemedicine guidelines (2005) also make a reference to the special interest group for telepathology clinical guidelines of ATA in 1999 [29].

The Canadian Association of Pathologists clinical guidelines on telepathology focuse only on WSI for intraoperative consultation/frozen-section primary diagnosis, expert or second opinions, and quality assurance practices $[7,9,10]$. This document includes the CAP recommendation for validation of digital pathology systems [10]. Since 2013, several WSI systems have received their Class II Medical Device License from Health Canada [30].

In the United States, the Food and Drug Administration (FDA) has declared that WSI systems are Class III devices and require premarket approval [30]. FDA has released a draft guide for technical specifications in digital pathology. Every component has two sections: description and test methods to evaluate them, and additional guidance or resources are also included. In a second part (system-level assessment), testing methods for image acquisition and display subsystems are described with sections for color reproducibility, spatial resolution, focusing, whole-slide tissue coverage and stitching errors. The final sections of the document are dedicated to user interface, labeling and quality control [19]. 
Table 1. Guidelines, position papers and directives related to WSI in pathology

Canada

Canadian Association of Pathologists (CAP-ACP)

2014: Guidelines from the Canadian Association of Pathologists for Establishing a Telepathology

Service for Anatomic Pathology Using Whole-Slide Imaging $[9,10]$

United States

College of American Pathologists (CAP)

2013: Validating Whole-Slide Imaging for Diagnostic Purposes in Pathology $[11,12]$

2011: Anatomic Pathology Checklist: CAP Accreditation Program [13]

American Telemedicine Association (ATA)

2014: Clinical Guidelines for Telepathology $[3,8,14,15]$

Digital Pathology Association (DPA)

2011: Validation of Digital Pathology in a Healthcare Environment [16]

2011: Archival and Retrieval in Digital Pathology Systems [2]

2011: Interoperability between Anatomic Pathology Laboratory Information Systems and Digital

Pathology Systems [17]

2011: Validation of Digital Pathology Systems in the Regulated Nonclinical Environment [18]

Food and Drug Administration (FDA)

2015: Technical Performance Assessment of Digital Pathology Whole-Slide Imaging Devices:

Draft Guidance for Industry and Food and Drug Administration Staff [19]

Centers for Medicare \& Medicaid Services (CMS)

2015: Clinical Laboratory Improvement Amendments (CLIA) [20]

Centers for Disease Control and Prevention (CDC)

2013: Clinical Laboratory Improvement Advisory Committee (CLIAC) [21]

Society of Toxicologic Pathology

2013: Validation of Digital Pathology Systems in the Regulated Nonclinical Environment [22]

2007: Pathology Position Paper on Pathology Image Data [23]

European Union

European Commission (EC)

2012: Guidelines on the Qualification and Classification of Stand Alone Software Used in

Healthcare within the Regulatory Framework of Medical Devices [24]

Spain

Spanish Society of Anatomic Pathology (SEAP-IAP)

2015: Practical Guidelines for Digital Pathology Implementation [25]

United Kingdom

The Royal College of Pathologists (RCP)

2013: Telepathology: Guidance from The Royal College of Pathologists [26]

Australasia

The Royal College of Pathologists of Australasia (RCPA)

2014: Position Statement: Telepathology [27]

The Clinical Laboratory Improvement Amendment Act (CLIA) from the Centers for Medicare and Medicaid Services imposes a series of procedures on about 251,000 laboratories in the United States, including the need to periodically validate digital pathology instruments and systems used for testing $[2,31]$.

The Centers for Disease Control and Prevention manages the Clinical Laboratory Improvement Advisory Committee (CLIAC, http://wwwn.cdc.gov/cliac/) to provide scientific and technical advice and guidance to the
US Department of Health and Human Services [21]. In the August 2013 meeting, digital pathology was discussed, with reference to WSI technology validation, color in medical imaging, health IT policy and standards, terminology and interoperability. CLIAC endorses the use of CAP guidelines [21].

The main objective of these guidelines is to demonstrate that internal validation is necessary to show that WSI is at least as effective as conventional microscopy for the specific purpose (e.g. routine primary diagnosis) [5, 32-35]. 
Regarding nonclinical (experimental/toxicological) anatomic pathology, the DPA white paper defined the technical factors to consider in the validation of digital pathology systems [22], and the Society of Toxicologic Pathology made recommendations on when validation is needed [36].

In the European Union, some WSI systems (both scanner and digital pathology software) have obtained a 'CE mark' and are classified as 'other' in vitro diagnosis medical devices. The declaration of conformity for these systems is based on the Directive 98/79/EC on in vitro diagnostic medical devices, and it does not include a performance evaluation [24].

The Spanish Society of Anatomic Pathology and the Spanish Division of the International Academy of Pathology has recently published clinical guidelines for digital pathology, including technical recommendations, procedures for digital slide validation (based on CAP and ATA guidelines), specimen traceability, image analysis and legislation and proposed a plan for image retention [25].

In 2013, the RCP released an update of the 2005 guidelines for the use and practice of telepathology, which included virtual slide applications. It is recommended that pathologists ensure that they have verified that they have the abilities and equipment required to make accurate diagnoses either by conventional or digital microscopy [26].

The position statement of The Royal College of Pathologists of Australasia expresses in two pages that diagnostic accuracy using virtual slides is comparable to conventional microscopy. It mentions the benefits of telepathology and the quality issues to be considered when telepathology is being assessed for provision of a diagnostic pathology service [27].

\section{Technical Considerations}

Before we evaluate technical aspects of a digital system, we should consider its main purpose, intended use (regulated or not regulated environment) and our main needs [22]. For instance, if we intend to use the digital slide system mainly for frozen-section teleconsultations, we need technical characteristics quite different from when our main idea is to replace a large volume of glass slides (if not all) to evolve towards a complete digital pathology workflow.

Existing digital pathology guidelines approve the use of WSI for clinical purposes, including primary diagnosis, expert opinion, intraoperative consultation, off-site diagnosis, education and quality assurance. ATA guidelines state that the medical director shall be responsible for em- ploying and validating these devices if they are to be used for non-FDA-approved applications [3]. Some authors consider that more data concerning specific details, like image resolution, color balance, data transmission, storage and degree of compression, are needed [7].

\section{Components of a Digital Slide System}

The components of a WSI system can be grouped in four main parts: the scanner (image acquisition), the image management (data and messages, including integration in LIS), the viewing software and the image storage system. An additional important component can be image evaluation and analysis systems $[3,22]$. These components will only work in an adequate hardware (e.g. user workstation and viewing monitor), software (e.g. operating system and user authentication) and network architecture [16].

Even when some recommendations are included for isolated components, we should remember that validation of a digital pathology system should be performed on the whole digital pathology system in toto [7], and assessing each component individually (e.g. scanners, servers and monitors) is only one part of the implementation process. However, current digital pathology systems are not truly 'closed' systems [28], and one part of the system (e.g. viewer and server) may be replaced, upgraded or updated [11].

According to the FDA, a digital pathology system must be accompanied by technical information on acquisition and image display (table 2) [19].

\section{Technical Recommendations for Digital Pathology Systems}

\section{Architecture}

The architecture of digital pathology systems can be based on client-server solutions, web applications or service-oriented architecture. In every case, it must be designed to be suited for all the personnel working in the pathology department, with access from any computer in the local network and adequate performance of the whole system (e.g. network bandwidth, database response time and the pathologist's workstation throughput), so that normal work pace is maintained or improved [25].

The system architecture must facilitate the incorporation of the digital slide system in the information systems employed in the pathology department and the 
Table 2. Technical information of a digital pathology system according to the FDA [19]

\begin{tabular}{|c|c|}
\hline Image acquisition & Image visualization \\
\hline From a glass slide, a digital slide file is obtained & $\begin{array}{l}\text { From a digital slide image, optical signals are created } \\
\text { for the human reader }\end{array}$ \\
\hline $\begin{array}{l}1 \text { Slide Feeder. It may be an independent component } \\
\text { from the scanner itself; the number and size of slides, } \\
\text { thickness tolerance (remember that most scanners } \\
\text { will not be able to scan repaired slides with double } \\
\text { glass) must be stated, as well as if the slide will keep } \\
\text { horizontal during all the process } \\
2 \text { Light Source. The type of lamp (halogen, xenon arc } \\
\text { or LED), model and estimated lifespan are important } \\
\text { information; regarding condenser, illumination } \\
\text { format (Köhler/critical), model and numerical } \\
\text { aperture are important technical details } \\
3 \text { Imaging Optics. The entire optic pathway must be } \\
\text { described, including microscope objectives } \\
\text { (manufacturer, type, magnification and numerical } \\
\text { aperture) and accessory lens(es); magnification, per } \\
\text { ISO 8039:1997 Optics and optical instruments } \\
4 \text { Mechanical Scanner Movement. Movements of the } \\
\text { stage or objectives; stage manufacturer, model, slide } \\
\text { retention mechanism, xyz movement resolution and } \\
\text { control must be known } \\
5 \text { Digital Imaging Sensor. Important information } \\
\text { includes type of sensor (CCD/CMOS), } \\
\text { manufacturer, dimensions in pixels, design, } \\
\text { responsivity, noise and readout rate (pixels or frames } \\
\text { per second) } \\
6 \text { Image Processing Software. Exposure control, white } \\
\text { balance, color correction, subsampling and any other } \\
\text { correction in pixel information must be included } \\
7 \text { Image Composition. In this section, scanning } \\
\text { method (linear, tiling or stitching) is described, } \\
\text { together with scanning speed for each magnification } \\
\text { and per tissue area, and z-axis scanning capabilities } \\
8 \text { Image File Formats. Compression method and } \\
\text { ration, file format, file organization (e.g. pyramidal } \\
\text { or folder with multiple files) and DICOM } \\
\text { compatibility are some important aspects }\end{array}$ & $\begin{array}{l}1 \text { Viewer. Image Review Manipulation Software } \\
\text { - Continuous x- and y-axis movement; buffering } \\
\text { adjacent areas ('prefetching') } \\
\text { - Continuous zoom (magnification) } \\
\text { - z-axis movement (multiple planes) } \\
\text { - Comparing several slides at the same time on } \\
\text { multiple windows } \\
\text { - Annotations or marks of areas of interest and } \\
\text { revisiting them } \\
\text { - Basic image manipulation (focus, color, white } \\
\text { balance, filters and histogram) } \\
\text { - Recording visited areas and magnification } \\
\text { - Multiple remote users can review the same area } \\
\text { (virtual 'multihead microscope') } \\
\text { 2 Computer Environment. Describing the type of } \\
\text { computer and its specifications in the pathologist } \\
\text { workstation, including: } \\
\text { - Type of computer (PC/Mac) } \\
\text { - Operating system (OS), 32 or } 64 \text { bit and version } \\
\text { (Windows 10, OS X 10.6, Linux/Ubuntu 11.10) } \\
\text { - Graphic card (manufacturer and model) } \\
\text { - Graphic card driver (manufacturer, model and } \\
\text { version) } \\
\text { - Color management settings [Intelligent Calibration } \\
\text { System (ICS) or Windows Color System (WCS)] } \\
\text { - Color profile (sRGB IEC61966-2.1) } \\
\text { - Display interface (DVI/DisplayPort) } \\
\text { 3 Monitor. A complete description of the display, } \\
\text { graphic cards and control software are recommended: } \\
\text { mainly: } \\
\text { - Display technology (LED, LCD) } \\
\text { - Real visualization, physical area } \\
\text { - In LCD displays, backlight type } \\
\text { - Pixel pitch (distance between points), pattern } \\
\text { - Subpixel or color driving techniques } \\
\text { - Video bandwidth } \\
\text { - OSD (on-screen display) controls } \\
\text { - Ambient light sensors } \\
\text { - Touch screen technology } \\
\text { - Color calibration and management tools } \\
\text { - Quality control procedures }\end{array}$ \\
\hline
\end{tabular}

health system (e.g. LIS, patient database and clinical records) [25].

\section{Communication Network}

Networks used for WSI should transmit images within the required timelines without resulting in image distortion and/or degradation [10].

International Clinical Guidelines for Digital Pathology
A typical image stream comprises about $5-20 \%$ of the whole-slide data, and the digital pathology systems are designed to balance the speed of the stream, efficient use of the network bandwidth and image quality. The hospital will need to dedicate at least 175 megabits per second (Mbps) network capacity for digital slide ingestion. A dedicated 1 gigabit per second (Gbps; Gigabit Ethernet) 
full duplex connection between the scanner and the online archive of the digital pathology system would ensure high ingestion performance $[2,25]$.

Within the hospital local area network, at least a 100Mbps network from the digital slide storage server to the workstation is recommended [2].

In a wide area network, the minimally required bandwidth for an acceptable viewing performance is $2 \mathrm{Mbps}$ [2].

\section{Interoperability: Integration in Other \\ Between-Information Systems}

The pathology imaging system should be integrated in other clinical systems, such as LIS via accepted standards such as HL7, as recommended by ATA [3], Canadian [10] and European guidelines [25, 37].

Digital pathology systems also store, retrieve, display and manage metadata associated with digital slides. At the scanner level, metadata such as the macroimage, scan parameters, scan plan and tissue maps are generated and associated with the image. When the digital pathology system is integrated in an LIS, then patient and histology (part/block/stain) data can also be retrieved from the LIS [2].

Data and image management software usually acts as an interface between the LIS and the digital pathology system. This software can also be configured to reflect the appropriate workflows [10]. Data transmission, integration, security and software performance should comply with existing CAP checklists, CLIA $[2,20]$ and Part 11 regulations for electronic records in the United States [23].

For the functional integration of the LIS and the digital pathology system, two scenarios are described in the DPA guidelines: (a) case reviewing is based on the digital imaging system that queries the LIS for additional information, or (b) case reviewing and worklist are managed by the LIS, and a digital imaging system is only used to review images and if annotations or quantitative analyses are performed; these data are transferred back to the LIS $[16,17]$.

Ideally, the digital pathology system should integrate not only the LIS and the clinical record, but also the hospital medical image system called PACS (Picture Archiving and Communication System), so that radiology, dermatology as well as pathology images from a clinical episode can be retrieved when needed [25]. PACS provides standard archival, retrieval and communication procedures for digital images [28], and they should follow the standards defined by Digital Imaging and Communi- cations in Medicine (DICOM) [7, 25], which are also available for pathology, although, so far, only few publications on experience have become available [38-41]. Test reports created and stored in a pathology PACS are subject to the same regulatory requirements as those created and stored in LIS [28].

The international standards that a digital pathology system should follow are $[10,17,37]$ :

- IHE (Integrating Healthcare Enterprise): Anatomic Pathology Technical Framework [42]

- DICOM Supplement 122: Specimen Module and Revised Pathology SOP Classes [43]

- DICOM Supplement 145: Whole Slide Microscopic Image IOD and SOP Classes [44]

\section{Scanning Speed}

Currently, the scanning time for a $15 \times 15$-mm specimen ranges from 2 min using a $\times 20$ objective to up to 10 min using a $\times 40$ objective [10]. We must include not only the time to capture the glass slide image, but also rack and slide loading, calibration, white balance, image processing and compression, and image transmission and storage. Therefore, the time that it takes from slide uploading to image viewing is the time that must be evaluated, especially for frozen-section or intraoperative studies $[25,45]$.

In a reasonably sized pathology department, a scanner could be used all day to generate up to 500-1,000 slides per day. Scanning speed can be affected by image compression or processing, network bandwidth and storage system, since all of them must be able to work at least as fast as the scanner $[2,25]$.

\section{Storage}

The pathology department should define processes and procedures for short- and long-term image storage, and accurate and timely retrieval of images [3]. Storage capacity for images and data should be at least enough to avoid loss of any data.

When needed (generally due to high costs), legislation allows the possibility to maintain the clinical information in a format different to the original [25]. In histopathology, the original specimen is usually a glass slide, resulting in data files several orders of magnitude larger than digital radiology images, and the pathology images must be digitized and compressed before transmission, with consequent possible loss of quality [26].

The image file associated with a $0.5-\mu \mathrm{m} /$ pixel $(\times 20)$ scan of a $15 \times 20$ - $\mathrm{mm}$ tissue specimen is as large as $3.6 \mathrm{~GB}$ and $\mathrm{a} \times 40$ scanned image can be as large as $14.5 \mathrm{~GB}$. The resulting images need to be compressed to more manage- 
able sizes (25:1 compression or greater). Typically, a 0.5$\mu \mathrm{m} /$ pixel $(\times 20)$ digital image can be stored in a JPEG2000 compression file of $200-500 \mathrm{MB}$. A similar $\times 40$ image could be stored in a JPEG2000-compressed file of $>600$ MB [2, 3]. Reversible (lossless) or irreversible (lossy) image compression may only be used to reduce the digital slide size if the resulting quality is sufficient to reliably perform the clinical task [3].

The DPA guidelines recommend redundant storage systems. If the image size is $200 \mathrm{MB}$, then a 1-TB disk storage holds about 3,500 digital slides when configured in redundancy like RAID 6 [2].

Two storage approaches are: storing every single image or selective image storage. The easiest solution is to store every single image used for patient health care regardless of its perceived diagnostic importance. In case this is not possible or necessary, selective image retention strategies are used. The criteria for this selection are still under debate. A possible recommendation is that newer, more frequently accessed cases be stored using rapid access technology (hot storage), while older cases can be archived for slower retrieval (cold storage) [7].

Storage of the digital slides near the scanner and histology laboratory is preferable, since images/data from its source location can be streamed to any external location [2].

\section{Image Quality in WSI}

Although there are no defined minimum technical standards in telepathology for image capture, storage, transmission or viewing at present, RCP guidelines include image resolution, the color depth to be captured, the speed with which images should be transferred from one site to another and viewing station characteristics [26].

Spatial image resolution should be measured in microns per pixel and not by the magnification index $(\times 20$ or $\times 40)$. A digital slide scanning system should be able to generate image resolutions of $0.5 \mu \mathrm{m} / \mathrm{pixel}$ (effective viewing magnification: $\times 20$ ) or better $[2,7,25]$. Some scanners use Plan-Apochromat $\times 20$ objectives with 0.8 numerical aperture, yielding images with $0.32 \mu \mathrm{m} /$ pixel [46].

Not only in cytology but also in some biopsies, 0.275 $\mu \mathrm{m} /$ pixel (effective viewing magnification: $\times 40$ ) may be needed with HE staining for prostate, renal and liver biopsies including special histochemical stains for organisms, such as an acid-fast and Helicobacter pylori stains $[34,35]$.

Some scanners are not able to scan immunohistochemistry slides with limited tissue and little or no posi- tive immunoperoxidase staining since they may fail to find the plane of focus for those slides even with manual selection of the scanning areas and focus points [35].

Using $\times 20$ magnification $(0.46 \mu \mathrm{m} /$ pixel $)$ and following CAP guidelines, Thrall et al. [35] concluded that WSI alone with no reference to glass slides, for the set of challenging cases they selected, could be problematic. These authors recommended establishing a standard for the level of performance decrease which is 'acceptable' in WSI compared to glass slides for primary diagnostic purposes.

The percentage of slides that need to be rescanned should not be greater than $5 \%$, although this figure may be directly related to the histotechnical procedure quality [25]. A poor histology technique (e.g. tissue folds, chatter artifacts from poor microtomy or mounting medium issues, such as dried mounting media with dirt on top of coverslips or air bubbles underneath coverslips) generates images that are inadequate for diagnostic purposes, and scanners may not be able to obtain adequate digital slides [10]. Automatic slide staining and coverslipping is highly recommended [25].

Some validation studies show rescanning rates greater than $13 \%$ during initial training, which fall to about $5 \%$ at the end of the training period [46]. In cytology, rescanning rates may rise to $10 \%$ [25].

\section{Image Compression}

Most guidelines state that image compression may be used to reduce digital slide size only if the resulting quality is sufficient to reliably perform the clinical task $[2,3$, 24-26]. When applying JPEG2000, a compression ratio between 20:1 and 32:1 does not seem to impact diagnosis $[47,48]$, although this figure may vary for different clinical applications [2, 25]. JPEG2000 is a highly efficient compression technique, with better image quality for a resulting smaller image file. However, it requires more computation power, and compression time may prolong scanning times $[25,49]$.

\section{Image Format}

Most guidelines do not make any reference to the file format in WSI. DICOM has not defined a standard format for digital slides either. The guidelines of the Spanish Society of Pathology recommend that the scanner should be able to generate universal file formats like TIFF or JP2 [25].

In the absence of established image standards or technical specifications for image capture, storage and transmission in diagnostic histopathology, there is no minimum requirement placed upon system suppliers and there is no guarantee of image quality [26]. 
Digital Slide Viewer and Human Interface

The digital slide viewer must be fast and with all the basic and advanced functionalities needed by the pathologist in the daily clinical work, including its integration in the image analysis system. Different functionalities are needed for different users (e.g. pathologists, technicians, other medical specialists and patients) [25].

Pathologists need a system that is able to load and navigate the images in 0-2 s [2]. Viewing software should support annotation, side-by-side viewing of multiple images [3] and, optionally, changing the appearance of each digital slide (e.g. flipping the slide into a virtual tray) once it has been viewed [46].

The response time of the human eye is of the order of $0.1 \mathrm{~s}$; hence, this is the response time for data (images) offered to the eye through a conventional microscope. A telepathology system that refreshes an image in $1 \mathrm{~s}$ is currently technically impressive but still much slower than a conventional microscope [26].

When reviewing old cases, pathologists may be satisfied if those prior images are available in 4-5 $\mathrm{min}$ [2]. Following CAP guidelines for validation, several studies found that slide review is performed 30\% more slowly with digital slides (181 s with a conventional microscope vs. $235 \mathrm{~s}$ for WSI, per case), although in these studies pathologists had little previous experience with WSI and studies are based on 60 cases or less for each pathologist $[34,35]$.

The digital pathology system should be able to create 'work lists' containing the cases that are going to be discussed and facilitate pathologist-clinician interactions in interdisciplinary meetings or for tumor boards or other reasons $[2,3,26]$. According to this information, images can be previously retrieved from slower storage media [2].

In order to achieve performance similar to that obtained while moving the slide around the microscope stage and focusing up and down using the conventional microscope after years or practice, converting this process to the computer screen requires both time and patience, and today there is no consensus on which is the best human interface device to control panning and zooming (e.g. mouse, 3DConnexion motion device or game controllers) $[34,46]$.

The viewer should be able to support multiple image formats and to open images from the hospital PACS [25].

\section{$z$-Axis (Multiplane or 3D) Scanning}

Usual histological sections have a width of 3-4 $\mu \mathrm{m}$ and usually they can be scanned in one single plane. In cytol- ogy and fluorescent in situ hybridization (FISH), in order to avoid high rescanning rates, multiplanar scanning is recommended $[25,50]$. The Canadian guidelines describe the current limitations of WSI with respect to multiplanar focusing in cytology $[7,10]$.

Scanning at $\times 40$ magnification with multiple $z$-axis planes may come very close to the performance of glass slides, even for the detection of $H$. pylori, and other (renal/prostate) biopsies may also benefit from it, although this means a significant increase in scanning time and image size [25, 34, 47].

In z-axis scanning, two options should be tested: (i) generating a $3 \mathrm{D}$ image with a stack of images that allows navigation in the $\mathrm{z}$-axis or (ii) the enhanced/extended focusing mode that selects the best focus in the z-axis for each tile and generates a single image [25].

\section{Monitor}

As a reference, studies with display devices having horizontal resolution of approximately 2,000 pixels $(2 \mathrm{~K})$, for instance, 1,920 × 1,080 pixel, 22-inch monitor, show that this is the minimum recommended for pathology diagnosis if basic viewer functionalities are needed [25]. The optimal viewing experience for a pathologist has been described with a 24 -inch monitor with $1,920 \times 1,200$ resolution and 0.27 pixel pitch, where the effective magnification of the images is balanced by the streaming performance $[2,51]$. CAP guideline validation studies using a screen size of $1,280 \times 1,084$ pixels (17- or 19-inch screen) have shown that some users ( $36 \%$ of the comments) complain of poor image quality at high magnification (e.g. concerns about finding organisms and distinguishing types of inflammatory cells), and $15 \%$ complain about poorly scanned slides, which were not well visualized even at low power [35].

Existing guidelines recommend that pathologists should determine whether or not an image, a viewer or a monitor is satisfactory to render a diagnosis $[3,10]$, and some specific technical recommendations are given:

- The diagonal dimension of the display distance should be about $80 \%$ of the viewing distance [3]

- The screen should be able to display the image at the originally acquired spatial resolution [3]

- Viewing devices should be color calibrated [3]

- Actual contrast (still images) should be 1,000-1,600:1 [25]

- Bightness of the display device should be with a luminance of 300 candela $(\mathrm{cd}) / \mathrm{m}^{2}$, or higher. 


\section{Mobile Devices}

ATA guidelines accept the use of mobile devices for telepathology 'as long as they can securely display the pathology image to be viewed at an acceptable level of quality', and IT personnel and users should be trained accordingly in security and privacy (e.g. audio muting, video muting, avoiding uncontrolled environment or activating remotely disabling options) [3]. Mobile computing systems, like an iPad tablet, have been evaluated following the CAP Pathology and Laboratory Quality Center guidelines. In a pilot study with a low number of cases $(n=61)$, compared to a widescreen-assisted digital workstation, an iPad tablet was found to be less confident for routine work flow, due to the small size of the monitor, but suitable for urgent cases [52].

Apple's iOS and Google's Android OS (operating system) together represent about $80-85 \%$ of the mobile market, but other platforms exist (e.g. Windows, Symbian and Blackberry). Regarding applications, although native apps may be able to achieve better performance, since they must be completely rewritten for each platform, HTML5 is an excellent option for cross-platform applications, and efficient HTML5-based WSI viewers are already available. Mobile applications for frozen-section WSI evaluation, pathology consultation and teledermatopathology, for example, are available [1].

\section{Security and Privacy}

For digital pathology systems, security or safeguards and privacy measures will need to be built for to comply with local, state, federal or international legislation, regulations, accreditation and ethical requirements, including proper storage of digital slides, metadata and information, such as annotations and analysis results, in order to ensure data integrity for the duration of image retention $[2,3,10,16,25]$.

Bar coding of slides is recommended since the bar code can be scanned and decoded by the whole-slide scanner and used by the digital pathology system to automatically associate the scanned image with the glass slide, previously accessed in LIS in the case context. The bar code is a unique identification for the digital image to ensure a valid electronic chain of custody [2].

\section{Conclusions}

Existing guidelines are mainly dedicated to telepathology and procedures to validate digital slide systems, and some technical information (e.g. file format and compression) is scarce. The FDA document on Technical Performance Assessment of Digital Pathology Whole Slide Imaging Devices is an excellent basis to understand the main specifications needed for each component of a whole-slide system, and future studies about the impact of technical specifications of isolated components and the entire system on specific clinical applications will be most useful to understand how existing clinical guidelines need to be adapted.

\section{References}

1 Hipp J, Bauer TW, Cornish TC, Dry SM, Evans AJ, Frishberg DP, Glassy EF, Hassell LA, Henricks WH, Pantanowitz L, Salama ME, Murphy D: CAP Pathology Resource Guide: Digital Pathology, version 5.0(1), Northfield, College of American Pathologists, 2014, http://www.cap.org/ (accessed August 8, 2015).

2 Chlipala E, Elin J, Eichhorn O, Huisman A, Krishnamurti M, Sabata B: Archival and Retrieval in Digital Pathology Systems. Madison, Digital Pathology Association, 2011, https://digitalpathologyassociation.org/_ data/files/Archival_and_Retrieval_in_Digital_pathology_Systems_final.pdf (accessed August 8, 2015).

3 Pantanowitz L (ed): Clinical Guidelines for Telepathology: August 2014. American Telemedicine Association, 2014, http://www. americantelemed.org/resources/telemedi- cine-practice-guidelines/telemedicine-practice-guidelines/clinical-guidelines-for-telepathology\#.VZj-_tEw-Uk (accessed August $8,2015)$.

4 Thorstenson S, Molin J, Lundström C: Implementation of large-scale routine diagnostics using whole slide imaging in Sweden: digital pathology experiences 2006-2013. J Pathol Inform 2014;5:14, http://www.jpathinformatics.org/text.asp?2014/5/1/14/129452 (accessed August 8, 2015).

5 Ordi J, Castillo P, Saco A, Del Pino M, Ordi O, Rodríguez-Carunchio L, Ramírez J: Validation of whole slide imaging in the primary diagnosis of gynaecological pathology in a university hospital. J Clin Pathol 2015;68:3339.

6 Stathonikos N, Veta M, Huisman A, van Diest PJ: Going fully digital: perspective of a Dutch academic pathology lab. J Pathol Inform
2013;4:15, http://www.jpathinformatics.org/ text.asp?2013/4/1/15/114206 (accessed August 8,2015$)$.

7 Hanna MG, Pantanowitz L, Evans AJ: Overview of contemporary guidelines in digital pathology: what is available in 2015 and what still needs to be addressed? J Clin Pathol 2015; 68:499-505.

-8 Evans AJ, Krupinski EA, Weinstein RS, Pantanowitz L: 2014 American Telemedicine Association clinical guidelines for telepathology: another important step in support of increased adoption of telepathology for patient care. J Pathol Inform 2015;6:13, http://www.jpathinformatics.org/text.asp?2015/6/1/13/153906 (accessed August 8, 2015).

9 Têtu B: The Canadian Association of Pathology guidelines for establishing a diagnostic telepathology service using whole-slide imaging. Ann Pathol 2014;34:256-257.
International Clinical Guidelines for Digital Pathology 
-10 Bernard C, Chandrakanth SA, Cornell IS, Dalton J, Evans A, Garcia BM, Godin C, Godlewski M, Jansen GH, Kabani A, Louahlia S, Manning L, Maung R, Moore L, Philley J, Slatnik J, Srigley J, Thibault A, Picard DD, Cracower H, Tetu B: Guidelines from the Canadian Association of Pathologists for establishing a telepathology service for anatomic pathology using whole-slide imaging. J Pathol Inform 2014;5:15, http://www.jpathinformatics.org/text.asp?2014/5/1/15/129455 (accessed August 8, 2015)

11 College of American Pathologists: Validating whole slide imaging for diagnostic purposes in pathology. http://www.cap.org/web/ home/resources/cap-guidelines/currentcap-guidelines/validating-whole-slide-imaging-diagnostic-purposes (accessed August 8, 2015).

12 Pantanowitz L, Sinard JH, Henricks WH, Fatheree LA, Carter AB, Contis L, Beckwith BA, Evans AJ, Lal A, Parwani AV; College of American Pathologists Pathology and Laboratory Quality Center. Validating whole slide imaging for diagnostic purposes in pathology: guideline from the College of American $\mathrm{Pa}$ thologists Pathology and Laboratory Quality Center. Arch Pathol Lab Med 2013;137:17101722, http://www.archivesofpathology.org/ doi/full/10.5858/arpa.2013-0093-CP (accessed August 8, 2015).

13 College of American Pathologists: Anatomic Pathology Checklist: CAP Accreditation Program. Northfield, College of American $\mathrm{Pa}$ thologists, 2011, http://www.cap.org/apps/ docs/education/OnlineCourseContent/2011/ LAP/Resources/Checklists/AP.pdf (accessed August 8, 2015).

14 Pantanowitz L, Dickinson K, Evans AJ, Hassell LA, Henricks WH, Lennerz JK, Lowe A, Parwani AV, Riben M, Smith CD, Tuthill JM, Weinstein RS, Wilbur DC, Krupinski EA, Bernard J: American Telemedicine Association clinical guidelines for telepathology. J Pathol Inform 2014;5:39, http://www.jpathinformatics.org/text.asp?2014/5/1/39/ 143329 (accessed August 8, 2015).

15 Pantanowitz L, Dickinson K, Evans AJ, Hassell LA, Henricks WH, Lennerz JK, Lowe A, Parwani AV, Riben M, Smith CD, Tuthill JM, Weinstein RS, Wilbur DC, Krupinski EA, Bernard J: ATA clinical guidelines for telepathology. Telemed JE Health 2014;20:1049-1056.

16 Lowe A, Chlipala E, Elin J, Kawano Y, Long RE, Tillman D, Schwartz J, Parwani A: Validation of Digital Pathology in a Healthcare Environment. Madison, Digital Pathology Association, 2011, http://digitalpathologyassociation.org/_data/files/DPA-HealthcareWhite-Paper-FINAL_v1.0.pdf (accessed August 8,2015 ).

17 Ellin J, Haskvitz A, Premraj P, Shields K, Smith M, Stratman C, Wrenn M: Interoperability between Anatomic Pathology Laboratory Information Systems and Digital Pathology Systems. Madison, Digital Pathology Association, 2011, pp 1-10, https:// digitalpathologyassociation.org/_data/files/ Interoperability_Between_Anatomic_Pathology_Laboratory_Information_Systems and_Digital_Pathology_Systems.pdf (accessed August 8, 2015).

18 Cann J, Chlipala E, Ellin J, Kawano Y, Knight B, Long RE, et al: Validation of Digital Pathology Systems in the Regulated Nonclinical Environment. Madison, Digital Pathology Association, 2011, https://digitalpathologyassociation.org/_data/files/DPA_White_Paper_Final_-_2011-11-17.pdf (accessed August 8,2015$)$.

19 FDA. Technical Performance Assessment of Digital Pathology Whole Slide Imaging Devices. Draft Guidance for Industry and Food and Drug Administration Staff. US Department of Health and Human Services, Food and Drug Administration, Center for Devices and Radiological Health, 2015, http://www. fda.gov/downloads/medicaldevices/deviceregulationandguidance/guidancedocuments/ucm435355.pdf (accessed August 8, 2015).

20 Centers for Medicare \& Medicaid Services Clinical Laboratory Improvement Amendments (CLIA). https://www.cms.gov/Regulations-and-Guidance/Legislation/CLIA/ (accessed August 8, 2015).

21 US Department of Health and Human Services: Clinical Laboratory Improvement Advisory Committee. Summary Report. August 21-22, 2013. Atlanta, US Department of Health and Human Services, http://ftp.cdc. gov/pub/CLIAC_meeting_presentations/ pdf/CLIAC_Summary/cliac0813_summary. pdf (accessed August 8, 2015).

22 Long RE, Smith A, Machotka SV, Chlipala E, Cann J, Knight B, Kawano Y, Ellin J, Lowe A: Scientific and Regulatory Policy Committee (SRPC) paper: validation of digital pathology systems in the regulated nonclinical environment. Toxicol Pathol 2013;41:115-124, http://tpx.sagepub.com/content/41/1/115. long (accessed August 8, 2015).

23 Tuomari DL, Kemp RK, Sellers R, Yarrington JT, Geoly FJ, Fouillet XL, Dybdal N, Perry R; Society of Toxicologic Pathology: Society of Toxicologic Pathology position paper on pathology image data: compliance with 21 CFR Parts 58 and 11. Toxicol Pathol 2007;35:450-455.

24 European Commission, DG Health and Consumer, Directorate B, Unit B2 'Health Technology and Cosmetics': Guidelines on the Qualification and Classification of Stand Alone Software Used in Healthcare within the Regulatory Framework of Medical Devices. http://ec.europa.eu/health/medical-devices/ files/meddev/2_1_6_ol_en.pdf (accessed August 8, 2015).

25 García-Rojo M, Conde AF, Ordi J, Ruiz-Martín J, Corominas JM, Álvarez-Alegret R, Alfaro L, Gatius S, Matías-Guiu X, de Álava E, Ramírez J: Guía práctica para la implantación de la patología digital; in Guerra Merino I (ed): Libro Blanco de la Anatomía Patológica en España 2015. Vitoria, Sociedad Española de Anatomía Patológica, 2015, pp 247-278, http://www.seap.es/libros-blancos (accessed August 8, 2015).

26 Lowe J: Telepathology: Guidance from The Royal College of Pathologists, October 2013. London, The Royal College of Pathologists, 2013, http://www.rcpath.org/Resources/RCPath/Migrated\%20Resources/Documents/G/ G026_Telepathology_Oct13.pdf (accessed August 8, 2015).

27 The Royal College of Pathologists of Australasia (RCPA): Position Statement: Telepathology. Australia: March 2014. Royal College of Pathologists of Australasia, 2014, http://www. telehealth.co.nz/images/telehealth/standards/Telepathology.PDF.

28 Lange H: Digital pathology: a regulatory overview. LabMedicine 2011;42:587-591, http:// labmed.ascpjournals.org/content/42/10/587. full (accessed August 8, 2015).

29 Duftschmid G, Binder M, Wrba T, Dorda W, Pehamberger H: Richtlinien zur Planung und Realisierung telemedizinischer Anwendungen. Wien Klin Wochenschr 2005;117:673683, http://www.who.int/goe/policies/countries/aut_support_tele1.pdf (accessed August 8, 2015).

30 Cornish TC, McClintock DS: Medicolegal and regulatory aspects of whole slide imaging-based telepathology. Diagn Histopathol 2014;20:475-481.

31 Ghaznavi F, Evans A, Madabhushi A, Feldman M: Digital imaging in pathology: wholeslide imaging and beyond. Annu Rev Pathol 2013;8:331-359.

- 32 Pantanowitz L, Valenstein PN, Evans AJ, et al: Review of the current state of whole slide imaging in pathology. J Pathol Inform 2011;2:36, http://www.jpathinformatics.org/text. asp?2011/2/1/36/83746 (accessed August 8, 2015).

33 Brachtel E, Yagi Y: Digital imaging in pathology - current applications and challenges. J Biophotonics 2012;5:327-335.

- 34 Buck TP, Dilorio R, Havrilla L, O’Neill DG: Validation of a whole slide imaging system for primary diagnosis in surgical pathology: a community hospital experience. J Pathol Inform 2014;5:43, http://www.jpathinformatics.org/text.asp?2014/5/1/43/145731 (accessed August 8, 2015).

- 35 Thrall MJ, Wimmer JL, Schwartz MR: Validation of multiple whole slide imaging scanners based on the guideline from the College of American Pathologists Pathology and Laboratory Quality Center. Arch Pathol Lab Med 2015;139:656-664, http://www.archivesofpathology.org/doi/10.5858/arpa.2014-0073-OA (accessed August 8, 2015).

36 Vahle JL: Introductory paper by Long et al on digital pathology validation. Toxicol Pathol 2013;41:114, http://tpx.sagepub.com/ content/41/1/114.full (accessed August 8, 2015).

37 Rojo MG, Daniel C, Schrader T: Standardization efforts of digital pathology in Europe. Anal Cell Pathol (Amst) 2012;35:19-23. 
38 Singh R, Chubb L, Pantanowitz L, Parwani A: Standardization in digital pathology: Supplement 145 of the DICOM standards. J Pathol Inform 2011;2:23, http://www.jpathinformatics.org/text.asp?2011/2/1/23/80719 (accessed August 8, 2015).

-39 Daniel C, Booker D, Beckwith B, Della Mea V, García-Rojo M, Havener L, Kennedy M, Klossa J, Laurinavicius A, Macary F, Punys V, Scharber W, Schrader T: Standards and specifications in pathology: image management, report management and terminology. Stud Health Technol Inform 2012;179:105-122.

40 Tuominen VJ, Isola J: Linking whole-slide microscope images with DICOM by using JPEG2000 interactive protocol. J Digit Imaging 2010;23:454-462.

-41 Zwönitzer R, Kalinski T, Hofmann H, Roessner A, Bernarding J: Digital pathology: DICOM-conform draft, testbed, and first results. Comput Methods Programs Biomed 2007;87:181-188.

42 IHE: Integrating Healthcare Enterprise: Anatomic Pathology Technical Framework. http:// www.ihe.net/ Technical_ Frameworks/\#anatomic (accessed August 8, 2015).

43 DICOM Standards Committee, Working Groups 26, Pathology: Digital Imaging and Communications in Medicine (DICOM). Supplement 122: Specimen Module and Revised Pathology SOP Classes. Rosslyn, DICOM, 2008, ftp://medical.nema.org/medical/ dicom/final/sup122_ft2.pdf (accessed August $8,2015)$.
44 DICOM Standards Committee, Working Group 26, Pathology. Digital Imaging and Communications in Medicine (DICOM). Supplement 145: Whole Slide Microscopic Image IOD and SOP Classes. Rosslyn, DICOM, 2010, ftp://medical.nema.org/medical/ dicom/final/sup145_ft.pdf (accessed August $8,2015)$.

45 Ribback S, Flessa S, Gromoll-Bergmann K, Evert M, Dombrowski F: Virtual slide telepathology with scanner systems for intraoperative frozen-section consultation. Pathol Res Pract 2014;210:377-382.

46 Jones NC, Nazarian RM, Duncan LM, Kamionek M, Lauwers GY, Tambouret RH, Wu CL, Nielsen GP, Brachtel EF, Mark EJ, Sadow PM, Grabbe JP, Wilbur DC: Interinstitutional whole slide imaging teleconsultation service development: assessment using internal training and clinical consultation cases. Arch Pathol Lab Med 2015;139:627-635, http:// www.archivesofpathology.org/doi/10.5858/ arpa.2014-0133-OA (accessed August 8, 2015).

47 Kalinski T, Zwönitzer R, Sel S, Evert M, Guenther T, Hofmann H, Bernarding J, Roessner A: Virtual 3D microscopy using multiplane whole slide images in diagnostic pathology. Am J Clin Pathol 2008;130:259-264, http:// ajcp.ascpjournals.org/content/130/2/259. long (accessed August 8, 2015).

48 Krupinski EA, Johnson JP, Jaw S, Graham AR, Weinstein RS: Compressing pathology whole-slide images using a human and model observer evaluation. J Pathol Inform 2012; 3:17, http://www.jpathinformatics.org/text. asp?2012/3/1/17/95129 (accessed August 8, 2015).
9 Lejeune M, López C, Bosch R, Korzyńska A, Salvadó MT, García-Rojo M, Neuman U, Witkowski L, Baucells J, Jaén J: JPEG2000 for automated quantification of immunohistochemically stained cell nuclei: a comparative study with standard JPEG format. Virchows Arch 2011;458:237-245.

50 Wright AM, Smith D, Dhurandhar B, Fairley T, Scheiber-Pacht M, Chakraborty S, Gorman BK, Mody D, Coffey DM: Digital slide imaging in cervicovaginal cytology: a pilot study. Arch Pathol Lab Med 2013;137:618-624.

51 Randell R, Ambepitiya T, Mello-Thoms C, Ruddle RA, Brettle D, Thomas RG, Treanor D: Effect of display resolution on time to diagnosis with virtual pathology slides in a systematic search task. J Digit Imaging 2015;28: 68-76.

52 Brunelli M, Beccari S, Colombari R, Gobbo S, Giobelli L, Pellegrini A, Chilosi M, Lunardi M, Martignoni G, Scarpa A, Eccher A: iPathology cockpit diagnostic station: validation according to College of American Pathologists Pathology and Laboratory Quality Center recommendation at the Hospital Trust and University of Verona. Diagn Pathol 2014; 9(suppl 1):S12, http://www.diagnosticpathology.org/content/9/S1/S12 (accessed August $8,2015)$. 